RECYT

Año 23 / № 35 / 2021 / 22-29

\title{
Asociación de realidad aumentada y aeronaves no tripuladas: investigaciones y oportunidades emergentes en AECO
}

\author{
Association of augmented reality and unmanned aircraft: emerging research and \\ opportunities in AECO
}

\section{Associação de realidade aumentada e aeronaves não tripuladas: pesquisas emergentes e oportunidades em AECO}

\author{
João Paulo Franco Assumpção*, Ana Regina Mizrahy Cuperschmid \\ Universidad Estatal de Campinas (Unicamp). Brasil. \\ *E-mail: j229322@dac.unicamp.br \\ Recibido: 18/12/2020; Aprobado 22/03/2021
}

\section{Resumen}

\begin{abstract}
En vista del difícil acceso a áreas remotas, edificios, sitios y sitios arqueológicos, las aeronaves no tripuladas (UA), aparecen como una alternativa menos invasiva para obtener información in loco. El uso de UAs ha sido recurrente en el área de Arquitectura, Ingeniería, Construcción y Operación (AECO) debido a la facilidad de uso y la posibilidad de integración con los más diversos sensores (cámaras, GPS, proximidad, etc.), brindando a los usuarios una nueva perspectiva del proyecto. Otra tecnología que se ha explorado en AECO es la Realidad Aumentada (AR), ya que permite la inserción de elementos virtuales en el entorno físico en tiempo real. Considerando las posibilidades de estas tecnologías, este artículo tiene como objetivo mapear investigaciones que buscan el uso simultáneo de UA y RA y discutir las oportunidades que esta asociación ofrece para AECO. Para el mapeo, se realizó una Revisión Sistemática. Solo se identificaron algunas investigaciones en el área de AECO, lo que indica un campo de conocimiento aún poco explorado. El aporte de esta investigación es investigar el uso de la RA junto con los UAS aplicados a AECO, destacando las brechas en el tema y señalando tendencias y oportunidades para los procesos de diseño, construcción, mantenimiento y operación de edificios.
\end{abstract}

Palabras clave: Realidad Aumentada; Aeronaves no tripuladas; Drone; AECO.

\section{Abstract}

Considering the difficult access to remote areas, buildings, places and archaeological sites, unmanned aircrafts (AUS), also known as drones, emerge as a less invasive alternative to obtaining information from such sites. The employment of UAs has been recurrent in the field of Architecture, Engineering, Construction and Operation (AECO) due to ease of use, access to previously unreachable areas and the possibility of integration with the most diverse sensors (cameras, GPS, proximity, etc.), giving users a new perspective of the project. Another technology explored in AECO is Augmented Reality (AR) because it allows the insertion of virtual elements in the physical environment in real time. Considering the possibilities of these technologies, this article maps research that seeks the simultaneous use of UAs and AR and discuss the opportunities that this association offers for AECO. For mapping, a Systematic Literature Review was conducted. Only a few studies were identified in AECO, indicating a field of knowledge that should be explored. This research contribution is the investigation of the emerging research about AR with UAS applied to AECO, highlighting the gaps in the theme and the opportunities for building design, construction, maintenance, and operation processes.

Keywords: Augmented Reality; Unmanned Aircraft; Drone; AECO.

\section{Resumo}

Tendo em vista o difícil acesso a áreas remotas, construções, locais e sítios arqueológicos, as aeronaves não tripuladas (unmanned aircrafts, UAs), também conhecidas como drones, surgem como uma alternativa menos invasiva para obter informações de tais locais. A utilização de UAs tem sido recorrente na área de Arquitetura, Engenharia, Construção e Operação (AECO) devido a facilidade de uso e a possibilidade de integração com os mais diversos sensores (câmeras, GPS, proximidade etc.), dando aos usuários uma nova perspectiva do projeto. 
Outra tecnologia que tem sido explorada em AECO é a Realidade Aumentada (RA), pois possibilita a inserção de elementos virtuais no ambiente físico em tempo real. Considerando as possibilidades dessas tecnologias, este artigo tem por objetivo mapear as pesquisas que buscam o uso simultâneo de UAs e RA e discutir as oportunidades que esta associação oferece para AECO. Para o mapeamento, foi realizada uma Revisão Sistemática da Literatura (RSL). Foram identificadas somente algumas pesquisas na área de $A E C O$, indicando um campo de conhecimento ainda pouco explorado. A contribuição desta pesquisa está em investigar a utilização de RA juntamente com UAS aplicada à AECO, evidenciando as lacunas do tema e apontando as tendências e oportunidades para os processos de projeto, construção, manutenção e operação de edificações.

Palavras-chave: Realidade Aumentada; Aeronave não tripulada; Drone; AECO.

\section{Introdução}

As aeronaves não tripuladas, conhecidas por Unmanned Aircrafts (UAs), datam desde fins do século XIX [1]. As tecnologias para as UAs vêm evoluindo desde então, permitindo sistemas de controle mais precisos e acurados e o acoplamento de recursos para os mais variados fins como agricultura, militar e construção civil.

Tendo em vista o difícil acesso a áreas remotas, construções, locais e sítios arqueológicos, as UAs, também conhecidas como drones, surgem como uma alternativa menos invasiva para obter informações de tais locais [2]. $\mathrm{O}$ uso de UAs tem sido recorrente na área de Arquitetura, Engenharia, Construção e Operação (AECO) devido a facilidade de uso, o acesso a áreas antes inalcançáveis e a possibilidade de uso dos mais diversos sensores (câmeras, GPS, proximidade, etc.).

Em AECO, os Unmanned Aircraft Systems (UAS) vêm sendo utilizados de diversas maneiras: fotogrametria aérea, possibilitando a restituição digital de edifícios a partir da técnica de Dense Stereo Matching (DSM) [3], [4]; visualização de modelos tridimensionais de edifícios em RA para análise e discussão do projeto [5], [6] e; na gestão e manutenção de edifícios, realizando uma varredura e auxiliando na identificação de problemas existentes [7] as well as the sustainability of critical healthcare infrastructure. The purpose of this paper is to propose a conceptual ambient intelligent environment for enhancing the decision-making process of the facility managers. This low-cost data-rich environment would use building information modeling (BIM.

Outra tecnologia que vem evoluindo desde a década de 1950 [8] é a Realidade Aumentada (RA). A RA consiste em um feedback natural ao operador com elementos simulados [9], isto é, o operador visualiza o mundo físico e em tempo real através de uma tela ao mesmo tempo em que interage com elementos virtualmente inseridos na cena.

A RA também vem sendo empregada na indústria AECO em ao menos três fases da construção: projeto, obra e operação. Na fase do projeto, a RA é utilizada para visualização in loco [6] discussão da forma, materiais construtivos e circulação [5], apoio ao projeto participativo [10] gerando uma visão composta (ambiente real com a adição de modelos virtuais. Na construção, a RA é utilizada na visualização e locação de equipamentos [11], [12] [13] Na fase de operação, auxilia na manutenção de facilities [14].

Uma interessante aplicação de RA para a visualização de edifícios pelo mercado imobiliário, foi a exibição do edifício Fibrasa Connection, realizado pela construtora Rossi em Vitória (ES) [15], [16]. Nessa ação publicitária, o projeto da edificação era visualizado in loco, em escala real, sobre um marcador de $900 \mathrm{~m}^{2}$, considerado o maior marcador para RA do mundo [17]. A construtora utilizou um helicóptero para propiciar aos possíveis investidores a visualização do edifício através de RA. Para tanto, foram realizados diversos testes de inserção do modelo no terreno e animações que visavam transmitir a sensação de realidade como de pessoas passeando pela calçada, carros transitando pela rua e o pouso de um helicóptero no heliponto do empreendimento [15].

A possibilidade de utilizar UAS para visualização em RA de edificações em tamanho real e in loco, representa uma alternativa mais acessível se comparada a um helicóptero, como o utilizado para visualizar o empreendimento Fibrasa Connection.

Com o intuito de mostrar pesquisas que relacionam as tecnologias de RA e UAS simultaneamente, este trabalho busca mapear as pesquisas recentes acerca desta temática por meio de uma Revisão Sistemática da Literatura (RSL), e aponta oportunidades de aplicação em AECO.

\section{Tecnologias emergentes: RA e UAS}

\section{Realidade Aumentada}

A RA era abordada em diversas publicações, no entanto, não havia a devida conceituação [9]. Isso porque não havia uma definição consistente a se considerar. Entendia-se como RA, projetos que consistiam em tecnologias baseadas em Telepresença e Telemanipuladores [9]. Posteriormente, surgiu um outro conceito mais restrito, que definia a RA como sendo uma RV em que se utilizava uma tela vestível de realidade aumentada (HMD) transparente, o que permitia uma visão clara do mundo real [9].

Entretanto, é impossível separar ambos os tópicos (RA e RV). Os dois possuem aspectos em comum. A RV é um ambiente totalmente sintético, no qual o operador está completamente imerso [9]. Esse ambiente virtual pode 
imitar características físicas do ambiente real, inclusive ser suscetível as leis da física como a gravidade. Deste modo, os autores apresentaram seu continuum (ou sequência contínua) entre Realidade e Virtualidade (Figura 1).

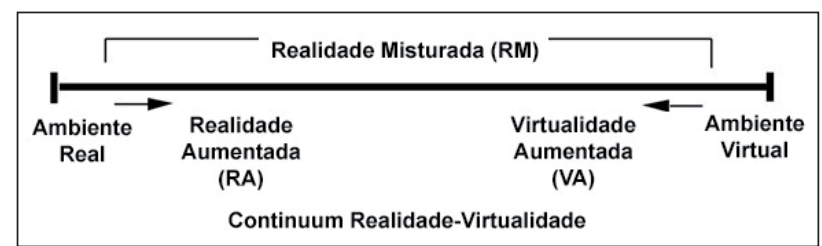

Figura 1: Sequência contínua entre Realidade e Virtualidade - baseado em [9].

Fonte: Elaborado pelos autores.

Esse continuum apresenta a esquerda da régua o Ambiente Real, composto por objetos reais visto através do mundo real [9] e em tempo real [18]. Esse ambiente real pode ser visualizado sem o auxílio de qualquer dispositivo, através de uma janela ou telas (monitoramento por vídeo). Do lado direito da régua, definiu-se o que seria o Ambiente Virtual. Ele consiste somente em objetos virtuais, desenvolvido por simulações gráficas e modelos tridimensionais gerados por computador, visualizados através de monitores ou telas de imersão. Tudo que se encontra entre o Ambiente Real e o Ambiente Virtual foi definido como sendo uma Realidade Misturada ou Mista (RM) [9]. A RM consiste em um ambiente que possui tanto elementos reais, quanto virtuais.

Os fatores que distinguem a RA vão muito além dos monitores [9]. Para os autores, a diferença estava em se a pessoa se encontrava imersa egocentricamente em um ambiente real ou observando o mundo real ao seu redor exocentricamente. A RA permite o usuário visualizar uma composição feita por objetos virtuais em um mundo real [19], [20]. Ela é capaz de criar conexões entre os dois mundos (real e virtual) através de uma interface simples e instantânea, aprimorando o mundo físico [20].

A interface de RA favoreceu novas possibilidades em relação a interação com os usuários, passando do tradicional gráfico bidimensional de telas planas para um tridimensional e interativo [8]. As principais características que definem a RA são: (i) combinar real com virtual; (ii) interação em tempo real e; (iii) possuir registro tridimensional [21]. Alguns exemplos desse uso na Arquitetura é a RA criada pela construtora Rossi para a publicidade de um novo empreendimento [15], [16] e o uso para análise de implantação do edifício [5], [6].

Por sua vez, a Virtualidade Aumentada consiste em um ambiente completamente digital, parcial ou totalmente imersivo e com o acréscimo de elementos reais [9]. Um exemplo dado pelos autores foi a inserção das mãos no ambiente virtual, com a possibilidade de interações como apontar e arrastar objetos. Um exemplo desse uso é um aplicativo que fornecia um modelo virtual da Torre Grossa (San Gimignano, Italy), no qual havia sensores posicionados nesse ambiente virtual que forneciam dados emitidos pelos sensores reais implantados in loco [2].

Kipper e Rampolla apresentam alguns componentes de hardware e software necessários para a construção de um ambiente de RA [19]. Os componentes de hardware apontados foram: computador de mesa ou dispositivo móvel; monitor ou tela; câmera; sensores de rastreio (GPS, magnetômetro, acelerômetro, etc.); estrutura de rede; marcador (elemento que auxilia o computador locar o objeto virtual no mundo real). Os componentes de software indicados foram: aplicativo que rode localmente; serviço web; servidor de conteúdo.

As principais vantagens da RA incluem a facilidade de colaboração, interação intuitiva, integração de informação digital e computação móvel [12]. Para os autores, a RA em AECO tem sido explorada para fins educacionais, de visualização de modelos e para interação em projetos colaborativos e participativos. Um exemplo é projeto de desenvolvimento de um parque colaborativo, no qual a partir da realidade aumentada é possível definir os equipamentos e a posição deles em um determinado parque [22].

Com a RA é possível vincular elementos virtuais e interfaces tangíveis para manipulá-los semelhantes a objetos reais [12].

O uso da Modelagem da Informação da Construção (Building Information Modeling - BIM) associado a RA, tem auxiliado na criação de modelos mais realistas para AECO [12]. É possível definir 5 técnicas fundamentais para um ambiente de RA realista [23]: (i) uma técnica de modelagem em que se visualiza as formas físicas; (ii) uma técnica de rastreio em que se integra visualmente ambientes reais e elementos virtuais; (iii) uma técnica de renderização que gere uma geometria suficientemente realista; (iv) uma técnica capaz de gerar uma saída baseada no banco de dados de um modelo BIM e; (v) uma técnica avançada de rastreio que envolva equipamentos e movimentos.

Para alcançar tal realismo é fundamental observar os graus de liberdade dos usuários. Uma RA pode ter até seis graus de liberdade (6DoF, six degrees of freedom), que se referem a posição e orientação da câmera dentro do espaço tridimensional [8]. Diz-se que a RA possui 6DoF quando há possibilidade de translação nos eixos $x, y$ e $z$, além de rotação (rodar, guinar e rolar). Alguns sistemas fornecem apenas três graus de liberdade (3DoF), sendo todos os três de rotação, Figura 2. 


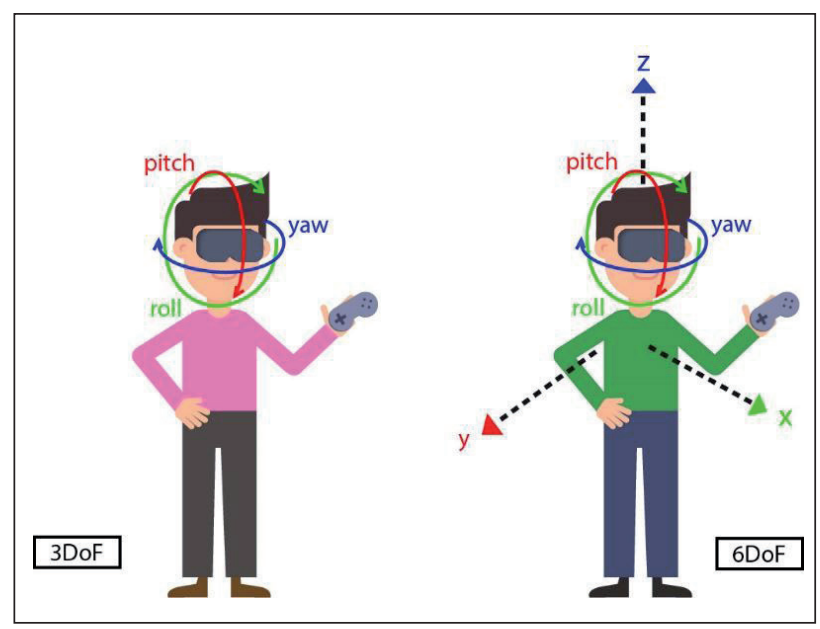

Figura 2: 3DoF e 6DoF.

Fonte: Elaborado pelos autores.

Tratando-se de RA, é importante determinar a posição e a orientação continuamente das entidades: cabeça, olhos ou membros. Isso está diretamente relacionado ao conceito de rastreamento, o qual visa obter as propriedades espaciais durante o tempo de execução [20]. Em outras palavras almeja "o registro correto e consistente entre os objetos virtuais e o ambiente real" [10] gerando uma visão composta (ambiente real com a adição de modelos virtuais.

UAS

Há três tipos de robôs móveis conforme o meio em que são utilizados: (i) terrestres; (ii) aquáticos e; (iii) aéreos. Os robôs móveis aéreos ficaram popularmente conhecidos como drones. Drone é uma palavra inglesa para zumbido. Portanto, esse nome é devido ao ruído comumente existente nesse tipo de robô e por serem voadores.

Neste artigo, adotou-se UA, por ser o termo utilizado pelo Departamento de Controle do Espaço Aéreo (DECEA) desde julho de 2020 [24]. Entretanto, a UA também é conhecida como Veículo Aéreo Não Tripulado (VANT) e aqueles que possuem 4 hélices também podem ser chamados de quadcopteros ou quadrotores. Quando se tratar do sistema, ou seja, da aeronave e de todos os componentes que envolvem o sistema (controle remoto, sistemas de transmissão de dados, HMD, etc.), utiliza-se o termo UAS.

A primeira aeronave não tripulada que se tem notícia, foi uma pipa com um sensor do tipo anemômetro -dispositivo utilizado para medir velocidade e direção dos ventoscolocada 1.200 pés de altura resultado de um experimento realizado pelo inglês Douglas Archibald em 1883 [1].

Com o advento de novas tecnologias digitais, permitindo maior acurácia nos voos realizados através de um UAS, a fabricante japonesa Yamaha iniciou a primeira fabricação de UAs em larga escala para uso civil [25]. Tratava-se de uma aeronave de hélice rotativa, denominada R50, utilizada na semeadura e pulverização de campos de arroz.

Atualmente, as UAs podem ser classificados da seguinte forma [26]: (a) Asas fixas - referindo-se a aviões não tripulados e que necessitam de uma pista para pouso e decolagem; (b) Asas ou hélices rotativas - corresponde às UAs capazes de decolar e pousar verticalmente como helicópteros; (c) Infláveis como balões e dirigíveis - são mais leves que o ar e voam em baixas velocidades e; (d) Batedores de asas - possuem asas com formatos orgânicos e flexíveis, tendo sua estrutura inspirada em insetos e aves.

\section{Uso simultâneo de RA \& UAS: pesquisas emergentes}

Com o intuito de mapear e avaliar criticamente as pesquisas emergentes sobre o uso de drones associado à RA na área AECO, foi realizado uma RSL. Essa RSL visa agregar os resultados de pesquisas acerca das tecnologias que têm sido utilizadas com a intenção de apoiar a visualização do projeto no ambiente construído em RA através de UAS, além de identificar lacunas a serem preenchidas com a proposição de trabalhos futuros [27]. Assim, esta RSL apresenta uma síntese evidenciando essas lacunas e apontando as tendências e oportunidades para o uso de RA associada a UAS em AECO.

\section{RSL: metodologia}

A questão que norteia esta RSL é saber o que vem sendo pesquisado sobre o uso de drones associado RA. Busca-se identificar como ocorre seu uso concomitante, quais engines ou sistemas são utilizados para misturar a imagem capturada pelo drone com elementos virtuais e os métodos de rastreio empregados. Para tanto, foi desenvolvido um framework conceitual (Figura 3).

A partir desta questão, definiu-se duas categorias: uma que englobasse as Realidades Misturadas e outra que indicasse o uso de UAs. A operação booleana AND foi aplicada entre essas categorias. Na primeira, foram utilizados os termos de busca "augmented reality" e "mixed reality". Na segunda, os termos "drone", "unmanned aerial vehicles" (veículo aéreo não tripulado), "remotely piloted aircraft" (aeronave pilotada remotamente), "quadrotor", "quadcopter" e "quadricopter". Todos os termos, dentro de cada categoria, foram separados pelo operador booleano OR.

Para se evitar "falsos positivos" nos resultados das buscas, optou-se por eliminar siglas, pois elas poderiam apresentar resultados com sentidos diferentes ao proposto na questão. Um exemplo de sigla, que retornaria resultados falsos, seria RPA que poderia apresentar tanto resultados contendo remotely piloted aircraft, quanto robotic process automation, sendo a segunda opção a mais encontrada durante uma busca com a sigla. Geralmente, o significado da sigla é apresentado no resumo ou nas palavras-chave.

O termo adotado por este trabalho "unmanned aircraft" não foi utilizado na busca, pois não apresentava resultados, uma vez que ele passou a ser adotado em julho de 2020 [24], após a busca as bases de dados. 


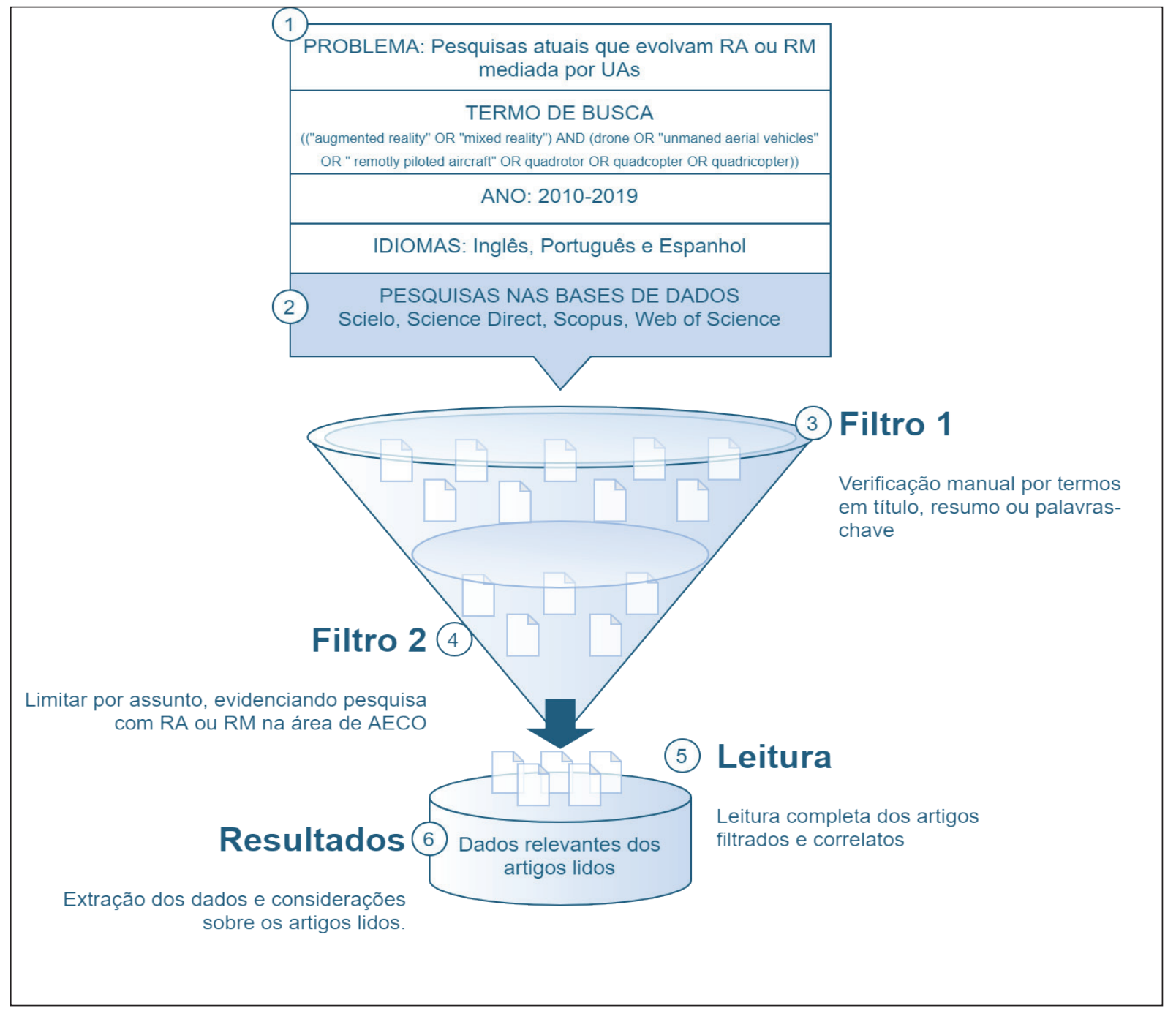

Figura 3: Framework conceitual.

Fonte: Elaborado pelos autores.

Adicionalmente, foram definidas algumas regras para a busca em relação ao período, ao idioma e ao tipo de publicação. Quanto ao tipo de publicação, dentre artigos em periódicos, artigos em eventos científicos, livros e capítulos de livros. Para garantir uma ampla busca por publicações, foram utilizadas quatro bases de dados conhecidas: Scielo, ScienceDirect, Scopus e Web of Science. Os termos das buscas foram limitados aos campos: título, resumo e palavras-chave.

\section{RSL: resultados}

A busca nas bases de dados gerou um total 247 publicações, sendo 8 através da ScienceDirect, 178 através da Scopus e 61 da Web of Science. A busca através da Scielo não retornou nenhum resultado (Figura 4).

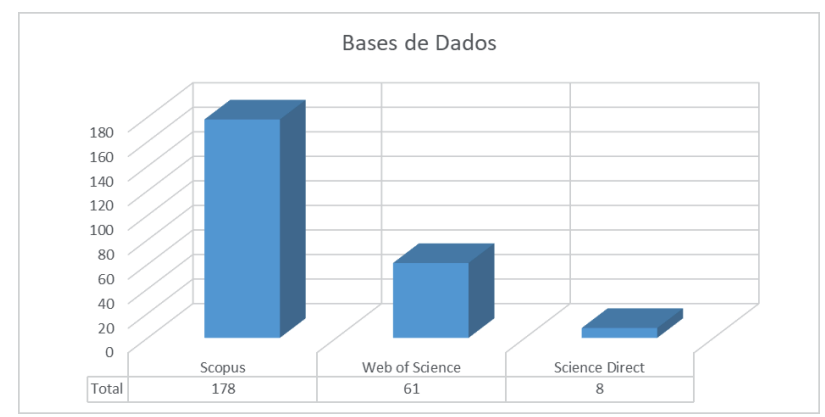

Figura 4: Base de dados pesquisadas. Fonte: Elaborado pelos autores.

A pesquisa nas bases de dado também mostrou um aumento nas publicações nos últimos anos, passando de 15 no ano de 2016, para $51 \mathrm{em} \mathrm{2017,70} \mathrm{em} 2018$ e $57 \mathrm{em}$ 2019 (Figura 5). 


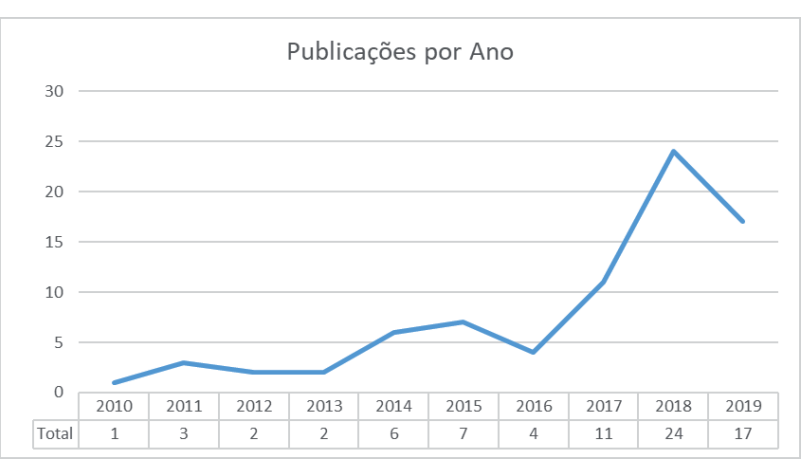

Figura 5: Artigos publicados por ano. Fonte: Elaborado pelos autores.

Após a busca, eliminou-se as publicações em duplicidade, ou seja, aquelas que foram encontradas em mais de uma base. Desta forma, 55 itens foram suprimidos do resultado da pesquisa, restando apenas 192 itens únicos.

Foi observado que alguns termos da pesquisa não estavam presentes no título, resumo ou palavras-chave da publicação. Portanto, foram verificados quais publicações realmente atendiam aos critérios de busca. Neste filtro, foram excluídas 113 publicações, restando agora 79 publicações únicas e que de fato atendiam aos termos da pesquisa (Figura 6).

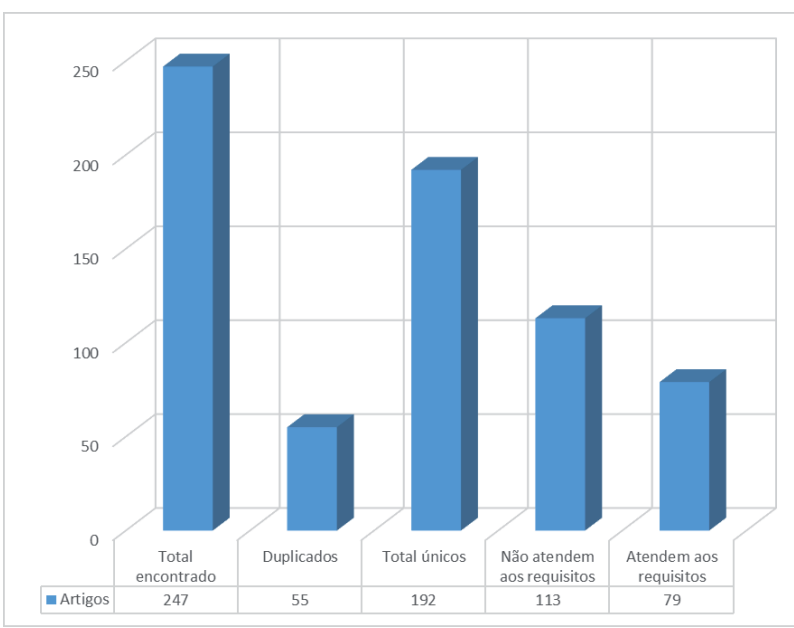

Figura 6: Seleção de resultados. Fonte: Elaborado pelos autores.

Quanto aos tipos de publicações, foram encontrados 61 artigos em eventos científicos, 17 artigos em periódicos e um capítulo de livro. Apenas um periódico apresentou mais de um artigo com os termos pesquisados, foi ele: SENSORS com 2 artigos publicados. Dentre as publicações em eventos científicos, seis se destacam com mais de duas publicações: ACM SIGGRAPH (5), International Conference on Human-Robot Interaction (4), International Conference on Virtual Systems and Multimedia (4), Conference on Human Factors in Computing Systems (4) (Figura 7).

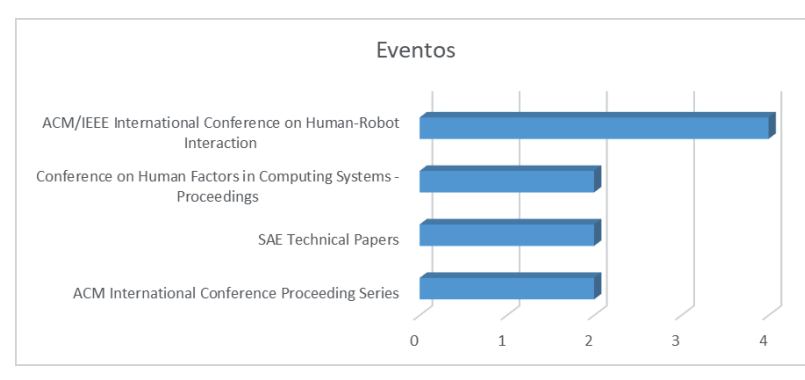

Figura 7: Artigo publicados em Anais de eventos. Fonte: Elaborado pelos autores.

Após uma lida nos 79 artigos restantes, eles foram classificados de acordo com o viés da pesquisa (Figura 8). A partir dessa leitura e classificação dos artigos, identificou-se seis artigos que tratavam sobre AECO. O termo AECO não pôde ser definido no termo da busca, pois a sigla representa quatro diferentes áreas com diversos sinônimos e a maioria das bases possuem limite de operadores booleanos. Esses artigos são apresentados nas discussões a seguir.

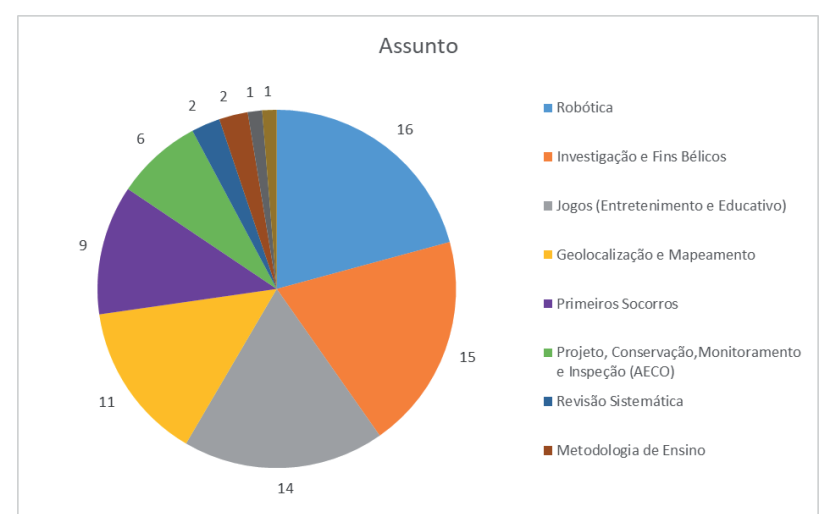

Figura 8: Artigos classificados de acordo com o viés. Fonte: Elaborado pelos autores.

\section{RSL: discussões}

Dos seis artigos que tratavam de Projeto, Conservação, Monitoramento e Inspeção com utilização de RA apoiada por UA, apenas quatro apresentaram os resultados do desenvolvimento de uma aplicação, os demais tratavam da idealização de um sistema.

Somente a aplicação de Wen e Kang [6] apresentou um modelo em escala e posição reais. Contudo, os autores utilizaram fotografias para definir a localização do modelo virtual, ou seja, fotografias como marcadores que ligam o modelo ao local.

Quatro pesquisas mencionaram terem utilizado a engine de jogos Unity para o desenvolvimento do aplicativo RA [2], [5], [6], [28].

Duas Interfaces de Programação de Aplicativos (Application Programming Interfaces, APIs) para desenvolvimento de aplicação em RA foram utilizadas nos artigos, são elas: Vulforia [6] Kudan [5], [28].

As UAs utilizadas e mencionadas pelos autores foram 
a Parrot AR.Drone 2 [5], uma DJI Inspire I [28], além de duas outras montadas pelas equipes de pesquisa: Micro Air Vehicle [2] e; uma UA feita em fibra de carbono contendo seis hélices e um controlador DJI [6].

Portanto, cada uma das pesquisas apresentou uma abordagem quanto ao método de rastreamento, exibição e interação, além de diferentes UAS e uso de APIs específicos. Os resultados indicam a necessidade de amadurecimento das tecnologias (tanto de hardware, quanto de software) para o maior aproveitamento de suas possibilidades em AECO.

\section{Tendências e oportunidades}

As diversas tecnologias de RA criadas permitiram que a indústria AECO utilizasse essas novas tecnologias na mesclagem entre o real e o projetado [12]. Desta maneira, conforme Schnabel e Wang, arquitetos, projetistas e engenheiros têm utilizado uma gama de instrumentos para alinhar o projeto idealizado ao mundo real [12]. Com a associação de RA com UAS, surgem novas oportunidades de aplicação em AECO.

Este estudo mapeou as pesquisas emergentes que buscam o uso simultâneo de RA e UAS através de uma RSL. Ao longo dos últimos cinco anos, foi possível observar o crescimento no número de artigos acerca da temática, sobretudo com a popularização das UAs, o desenvolvimento e a melhoria de sensores e técnicas de localização de robôs móveis.

A utilização de sensores para definição da localização do objeto virtual no mundo real carece de maior exploração. Sensores como o GPS e o IMU, imprescindíveis para a obtenção da odometria visual [29], merecem maior investigação quando se trata do uso simultâneo de RA e UAS. Observou-se uma lacuna no uso simultâneo de UAS e RA a partir de georreferenciamento. Tal associação permitiria a localização sem o uso de marcadores e a visualização do modelo no terreno em tempo real.

As oportunidades dessa associação incluem o fornecimento de diversas perspectivas do modelo em RA, que não poderiam ser obtidas por um dispositivo móvel nas mãos do usuário. Com isso, seria possível explorar possibilidades de implantação de uma edificação com maior liberdade e alcance. Tal associação tem potencial de auxiliar na verificação de erros de projeto, por proporcionar a visualização antecipada do projeto no local. Ainda, a organização dos canteiros de obra pode ser favorecida, reduzindo o desperdício e otimizando a produção. Adicionalmente, por meio de novos recursos tecnológicos, seria possível incluir outros participantes e permitir a interação coletiva com o modelo em RA.

Este artigo apresenta os recentes avanços nas pesquisas que tratam da associação de RA e UAS, identifica lacunas e aponta o potencial para a área de AECO, oferecendo direções para o desenvolvimento de novas pesquisas.

\section{Referências Bibliográficas}

1. P. G. Fahlstrom e T. J. Gleason, Introduction to UAV Systems, 4o ed. Chichester: Wiley, 2012.

2. D. Germanese et al., "Architectural heritage: $3 D$ documentation and structural monitoring using UAV”, in CEUR Workshop Proceedings, 2019, vol. 2320, p. $1-12$.

3. C. K. Cogima, R. V. C. Nascimento, P. V. V. Paiva, M. A. G. Carvalho, e E. Dezen-Kempter, "Scan-to-HBIM aplicado à igreja da Pampulha de Oscar Niemeyer", Gestão e Tecnol. Proj., vol. 15, no 1, p. 117-134, 2020.

4. E. Dezen-Kempter et al., "Historical District Information Modeling (HDIM): HBIM e SIG na documentação de sitios históricos", in Encontro Brasileiro de Modelagem da Informação da Construção e Patrimônio Cultural, 2020, p. 74-82.

5. S. Sreeram, K. K. Nisha, e R. Jayakrishnan, "Virtual Design Review and Planning Using Augmented Reality and Drones", in Proceedings of the 2nd International Conference on Intelligent Computing and Control Systems, ICICCS 2018, 2019, p. 915-918.

6. M.-c. Wen e S.-c. Kang, "Augmented reality and unmanned aerial vehicle assist in construction management”, in Proceedings of the 2014 International Conference on Computing in Civil and Building Engineering, 2014, p. $1570-1577$.

7. J. Irizarry, M. Gheisari, G. Williams, e K. Roper, "Ambient intelligence environments for accessing building information: A healthcare facility management scenario", Facilities, vol. 32, no 3, p. 120-138, 2014.

8. J. Peddie, Augmented Reality : Where We Will All Live, $1^{\circ}$ ed. Springer International Publishing, 2017.

9. P. Milgram, H. Takemura, A. Utsumi, e F. Kishino, "Augmented Reality: A class of displays on the reality-virtuality continuum", in SPIE Telemanipulator and Telepresence Technologies, 1995, vol. 2351, p. 282-292.

10. A. R. M. Cuperschmid, "Realidade Aumentada no processo de projeto participativo arquitetônico: desenvolvimento de sistema e diretrizes para utilização", Universidade Estadual de Campinas, 2014.

11. P. S. Dunston, J. v Sinfield, e D. Shin, "Spatial tracking challenge for Augmented Reality on building construction sites", in Proceedings of the 4th International Structural Engineering and Construction Conference, ISEC-4 - Innovations in Structural Engineering and Construction, 2008, vol. 2, p. 1247-1251.

12. M. A. Schnabel e X. Wang, orgs., Mixed reality in architecture, design and construction, $1^{\circ}$ ed. Sydney: Springer Science + Business Media, 2009.

13. G. Dini e M. D. Mura, "Application of Augmented Reality Techniques in Through-life Engineering Services", in Procedia CIRP, 2015, vol. 38, p. 14-23.

14. J. Irizarry, M. Gheisari, G. Williams, e B. N. Walker, "InfoSPOT: A mobile Augmented Reality method for accessing 
building information through a situation awareness approach", Autom. Constr., vol. 33, p. 11-23, 2012.

15. F. Martines, "A maior realidade aumentada do mundo - Link - Estadão”, Estadão, 2010. [Online]. Available at: https://link.estadao.com.br/noticias/geral,a-maiorrealidade-aumentada-do-mundo,10000043300. [Acessado: 06-ago-2020].

16. C. Yano, "Construtora cria maior projeto de realidade aumentada do mundo | Exame”, Exame, 2010. [Online]. Available at: https://exame.com/tecnologia/ construtora-cria-maior-projeto-realidade-aumentadamundo-574938/. [Acessado: 06-ago-2020].

17. Guinness World Records, "Largest augmented reality mark | Guinness World Records”, 30-jun-2010. [Online]. Available at: https://www.guinnessworldrecords.com/ world-records/largest-augmented-reality-mark. [Acessado: 12-ago-2020].

18. P. Lévy, Becoming Virtual: Reality in the Digital Age, 1o ed. New York: Plenum Trade, 1998.

19. G. Kipper e J. Rampolla, Augmented Reality: An Emerging Technologies Guide to AR. Elsevier Inc., 2012.

20. D. Schmalstieg e т. Hollerer, Augmented Reality: Principles and Practice (Usability). Pearson Education, 2016.

21. R. T. Azuma, "A Survey of Augmented Reality", Presence Teleoperators Virtual Environ., vol. 6, no 4, p. 355-385, 1997.

22. A. R. M. Cuperschmid, A. M. R. D. G. Monteiro, e R. C. Ruschel, "Desenvolvimento de aplicativo de realidade aumentada para uso em projeto participativo de áreas de lazer”, Eduacação Gráfica, vol. 20, no 3, p. 44-63, 2016.
23. J. w. Choi, "A Technological Review To Develop An ArBased Design Supporting System”, in Mixed Reality In Architecture, Design And Construction, X. Wang e M. A. Schnabel, Orgs. Dordrecht: Springer Netherlands, 2009, p. 53-74.

24. DECEA, Portaria n. 112/DGCEA (ICA 100-40) de 22 de maio de 2020. Instrução sobre aeronaves não tripuladas e o acesso ao espaço aéreo brasileiro. Brasília, DF: Departamento de Controle do Espaço Aéreo, 2020.

25. R. Austin, Unmanned Aircraft Systems: UAVs design, development and deployment, 10 ed. Wiltshire: Wiley, 2010.

26. K. Nonami, F. Kendoul, S. Suzuki, W. Wang, e D. Nakazawa, Autonomous Flying Robots: Unmanned Aerial Vehicles and Micro Aerial Vehicles, 1o ed. Springer Tokyo, 2010.

27. A. Dresch, D. P. Lacerda, e J. A. v. Antunes Jr, Design Science Research: método de pesquisa para avanço da ciência e tecnologia. Porto Alegre: Bookman, 2015.

28. L. Yan, T. Fukuda, e N. Yabuki, "Intergrating UAV development technology with augmented reality toward landscape tele-simulation", in Intelligent and Informed Proceedings of the 24th International Conference on Computer-Aided Architectural Design Research in Asia, CAADRIA 2019, 2019, vol. 1, p. 423-432.

29. F. Fraundorfer e D. Scaramuzza, "Visual Odometry : Part II: Matching, Robustness, Optimization, and Applications”, IEEE Robot. Autom. Mag., vol. 19, no 2, p. 78-90, 2012. 